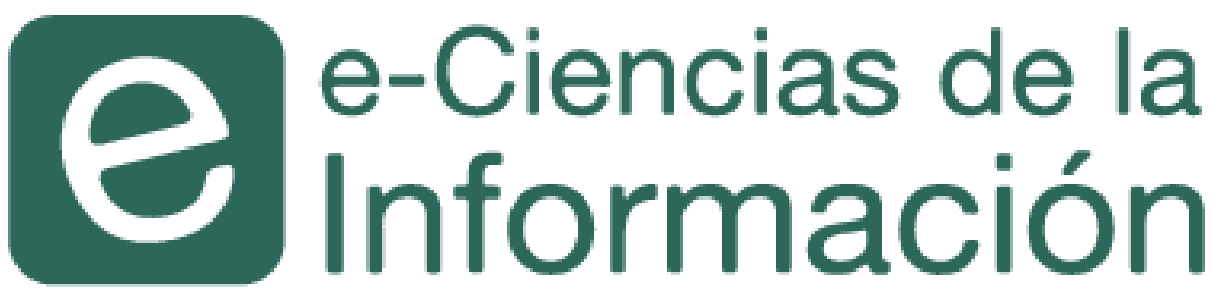

La biblioterapia como herramienta de ayuda aplicada en la biblioteca escolar: estudios de caso

Kimberly Naranjo Mora Cloriela Navarro Araya >atiana Zúñiga Seravalli

Publicado 01 de julio, 2017 / Artículo científico 2

\title{
(c) $\$(0)$
}

Escuela de Bibliotecología y Ciencias de la Información

Universidad de Costa Rica

Visite el sitio web de e-Ciencias de la Información 


\section{$>$ La biblioterapia como herramienta de ayuda aplicada en la biblioteca escolar: estudios de caso ${ }^{1}$}

Bibliotherapy applied as an aid tool in the school library: case studies

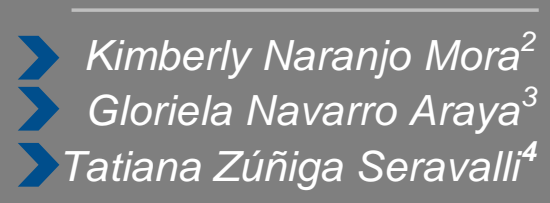

\section{RESUMEN}

Esta investigación se realizó con la finalidad de explorar la aplicación de servicios de biblioterapia en una biblioteca escolar pública costarricense, posicionando al bibliotecólogo como encargado principal, apoyado por un equipo de especialistas en otras áreas como educación, psicología, entre otros. Se enmarca en la metodología de investigaciónacción colaborativa, y pretende mediante la aplicación de la biblioterapia, trabajar con niños con problemas de conducta a nivel escolar, procurando mejorar en ellos la conducta, y promoviendo la cultura de paz mediante actividades lúdicocreativas cuyo principal eje es la lectura. Se realizó en una escuela costarricense, participaron seis niños, que acudieron a 19 intervenciones de biblioterapia grupales, con una duración de una hora cada intervención, durante un lapso de dos meses comprendidos entre los meses de setiembre, octubre y noviembre del año 2015. Se contó además con la asesoría dos profesionales especializadas en servicios de apoyo emocional. Es un aporte relevante para los profesionales en bibliotecología, pues les permite conocer, desde el punto de vista práctico, las fortalezas y debilidades de brindar un servicio de esta naturaleza, de manera tal que logren tomar decisiones con bases teóricas y mediante la aplicación práctica. Además, al ser una temática tan poco explorada en el ámbito nacional, permite explorar campos de acción nuevos para la profesión.

\section{ABSTRACT}

This research was conducted in order to explore the application of bibliotherapy services in a Costa Rican public school library, positioning the librarian as a leader, supported by a team of specialists in other areas such as education, psychology, among others. It is part of the research methodology collaborative action, and aims through the application of bibliotherapy, work with children with behavioral problems at school level, seeking to improve their behavior, and promoting the culture of peace through recreational and creative activities whose main shaft is reading. The research was conducted in a Costa Rican school in which six children participated in 19 library group interventions; each intervention lasted one hour within a two-month period on September, October and November in 2015. Moreover, the research was advised by two professionals that are specialists in emotional support services. It is an important contribution for professionals in librarianship, allowing them to know, from the practical point of view, the strengths and weaknesses of providing a service of this nature, so that reach decisions with theoretical basis and through practical application. In addition, being a subject so little explored at the national level, it can explore new fields of action for the profession.

\section{Recibido: 3 de octubre, 2016 | Aprobado: 3 de febrero, 2017}

1 Este artículo es producto del trabajo final de graduación de las autoras, titulado "La biblioterapia como herramienta de ayuda aplicada por el profesional de la información desde el marco de la biblioteca escolar: estudios de caso", defendido el 24 de mayo de 2016 en la Escuela de Bibliotecología de la Universidad de Costa Rica, para optar al título de Licenciatura en Bibliotecología y Ciencias de la Información.

2 Ministerio de Educación Pública. COSTA RICA. Orcid: orcid.org/0000-0003-0804-4246. kimnaranjom@gmail.com 3 Ministerio de Educación Pública. COSTA RICA. Orcid: orcid.org/0000-0003-4869-5852. glorielanavarroaraya@gmail.com 4 Municipalidad de San José. Sistema de Bibliotecas Municipales. COSTA RICA. Orcid: orcid.org/0000-0002-3414-1747. tatiana_punky@hotmail.es 
Palabras clave

Biblioterapia; Bibliotecas escolares; Educación para la paz; Educación de valores; Conducta; Comportamiento; Costa Rica.

\section{Keywords}

Bibliotherapy; School libraries; Peace education; Values education; Conduct; Behavior; Costa Rica.

\section{Introducción}

La bibliotecología es un campo profesional con una amplia gama de posibilidades donde la biblioteca, tal y como lo afirma Ferrada (2008), puede proveer servicios que contribuyan a la mejora de la calidad de vida de sus usuarios por lo que, desde una perspectiva académica, es menester conocer las bibliotecas a fondo y analizar su potencialidad para la práctica profesional, de tal manera que se puedan brindar otras opciones de inmersión en el campo laboral a los futuros profesionales y a los que están ejerciendo actualmente la profesión. En este sentido, el profesional en bibliotecología tiene como principio vital la búsqueda del bienestar y la mejora de los usuarios, por lo que una de las posibilidades para lograrlo es a través de la biblioterapia, sin embargo, el tema ha sido poco explorado a nivel nacional, razón por la cual, surge el interés por analizar y explorar su aplicación dentro de las unidades de información.

En este sentido, de acuerdo al Ministerio de Educación Pública (2012a) "el matonismo o bullying es la segunda situación violenta que ocurre con más frecuencia en los Centros Educativos, pues el $58,6 \%$ de los estudiantes así lo identifica" (p. 3). Por ello, en esta investigación se identificó como una posibilidad importante el estudio de la biblioterapia para identificar aquellas unidades en las que fuese realmente necesario el implementar actividades con su comunidad de usuarios dirigidos al comportamiento y valores relacionados a la paz.

Entonces, siendo evidente la necesidad por fomentar una cultura de paz y de sana conviviencia en el estudiantado escolar, se encontró la posibilidad de realizar este estudio sobre la biblioterapia desde el marco de la biblioteca escolar, para lograr el objetivo de contrarestar los efectos del bullying en la población escolar. De esta forma, se estableció la búsqueda de la mejora sus comportamientos con el apoyo de textos motivadores, que representen escenarios con los cuales quienes viven estas situaciones se identifiquen, para así crear una auto reflexión en el alumnado, en busca de una mejora continua en su actitud.

Por eso, la presente investigación tiene como objetivo explorar la aplicación de servicios de biblioterapia en una biblioteca escolar pública costarricense, posicionando al bibliotecólogo como encargado principal, apoyado por un equipo de especialistas en otras áreas como educación, psicología, entre otros.

De esta manera, seguidamente se presenta un desarrollo teórico sobre la aplicación de la biblioterapia dentro de la biblioteca escolar como herramienta para contrarestar los efectos del bullying, con el fomento de buenos valores, la sana convivencia y la práctica de una cultura de paz; así como también se explica la metodología de trabajo utilizada, los resultados de la investigación, su debida discusión y análisis para el desarrollo de las conclusiones obtenidas posteriormente. 


\section{Referente teórico}

Antes de aproximar la biblioterapia hacia la práctica en función de herramienta de ayuda para sopesar las diferentes características particulares presentes en la población de usuarios propios de la biblioteca escolar, es necesario comenzar por conocer y comprender qué es la biblioterapia, los diferentes tipos que existen, y los distintos campos en que ha sido aplicada, además del enfoque bibliotecológico.

Además, se conceptualizó y categorizó a los llamados problemas de conducta, para esclarecer de qué se tratan y los elementos que implica cada uno de ellos, y así, asociarlos posteriormente con la investigación, aplicados específicamente a los niños del estudio.

\subsection{Biblioterapia}

\subsubsection{Definición}

En términos básicos, la biblioterapia consiste en brindar apoyo emocional a un individuo por medio de los libros y la lectura, sin embargo, no se debe obviar que esta herramienta abarca y conlleva mucho más que lo señalado en la definición anterior, pues requiere de todo un método de aplicación por seguir para que produzca un efecto visible.

Autores como Tukhareli (2011), Toro (2010), Tomaino (2012), McCulliss (2012), Janavičienè (2010), Handbook of Non Drug Intervention Project Team (HANDI, 2013), y Gómez (2011) aportan diferentes conceptualizaciones del término biblioterapia. Entre ellas destacan:

"[Es] una terapia expresiva, que involucra el uso sistemático de libros para ayudarle a las personas a hacerle frente a sus problemas mentales, físicos, de desarrollo o sociales". (Tukhareli, 2011, p. 1)

Biblioterapia son [sic] las prácticas realizadas con textos literarios (lectura, narración, representación) como estrategia de intervención que impacte en la calidad de vida de los individuos, colaborando en la solución de situaciones difíciles o traumáticas de su historia y generando nuevos mecanismos para enfrentar y resolver problemas. (Tomaino, 2012, párr. 5).

"Proceso terapéutico, que se vale de textos determinados y lecturas dirigidas por el biblioterapeuta, que sirven como apoyo en el tratamiento de personas con alguna aflicción, con el propósito de provocar la catarsis y solución de problemas" (Gómez, 2011, p. 20). ${ }^{5}$

A partir de un exhaustivo análisis de los distintos conceptos del término que aportan otros autores y para los efectos de la investigación, la posición de las investigadoras hacia el concepto de biblioterapia se contempla como aquel proceso terapéutico por medio del cual se 
trabajan las particularidades emocionales y/o conductuales, pertenecientes ya sea a un individuo o a un grupo social peculiar, a través de la lectura guiada asociada a técnicas de animación lectora y de interacción social, y dirigida por un equipo profesional de las áreas de salud, educacional y bibliotecológica, con el objetivo primordial de ayudarles a lidiar con esos sentimientos y comportamientos, enfocándose en una actitud de mejora continua y positivismo. Como elementos importantes por tomar en cuenta dentro de la biblioterapia destaca la necesidad porque exista una interacción social constante entre quien facilita la herramienta y quien la recibirá, ya que la comunicación es un aspecto clave a la hora de proporcionar cualquier ayuda y para conocer realmente los resultados obtenidos durante el proceso de aplicación; además cabe subrayar el hecho de que la biblioterapia conlleva un trabajo conjunto por parte de diferentes profesionales pertenecientes al gremio de la salud, de la bibliotecología y de la educación, ya que cada uno de ellos aporta y examina detalles relevantes durante el proceso, sin embargo, el profesional de la bibliotecología será el líder durante el proceso, pues es este profesional quien conoce y comprende plenamente sobre los libros, documentos y técnicas de trabajo con respecto a la lectura, que son los elementos que giran en torno a la biblioterapia.

Otro factor importante de la biblioterapia es la necesidad del uso de textos que correspondan a las vivencias propias de las personas a las cuales se intenta ayudar por medio de esta herramienta, pues es de vital importancia vincular al participante directamente con la lectura, produciendo una reflexión interna sobre sí mismos que les permitan expresarse con libertad y así solucionar la problemática que afrontaba gracias a la interiorización de ese texto.

También es importante utilizar técnicas de apoyo, para que se dé un nivel de expresividad y comodidad mayor por parte de quien se somete a la biblioterapia, por ejemplo el dibujo, la dramatización, el juego lúdico, el uso de material audiovisual, la música, las manualidades, entre otras formas que potencien la exteriorización de los sentimientos y emociones del participante, de modo tal que la biblioterapia, además de brindar una lectura dirigida a la persona, le permita también manifestarse abiertamente sobre los temas tratados en el texto en cuestión.

\subsubsection{Tipología}

Al hablar sobre la biblioterapia es importante, en primera instancia, analizar cuáles son los objetivos por lograr con su utilización, el contexto en el cual se aplicará y, por supuesto, el perfil que presentan las personas a quienes se someterá a la biblioterapia como herramienta de ayuda.

Diferentes autores consultados sobre la temática de biblioterapia, tales como Morandi (2008), McCulliss (2012), Janavičienè (2010), Salinas (2009), Silva (2011), entre otros, difieren sobre los términos bajo los cuales clasifican los tipos de biblioterapia existentes, sin embargo coinciden en las características que presentan cada uno de ellos, por lo que se tomó como base principal la dispuesta por Daiva Janavičienè (2010): "la biblioterapia clínica, la biblioterpia rehabilitacional y la biblioterapia educacional" (p. 122).

- Biblioterapia clínica: Usualmente es utilizada en instituciones en las que se atienden pacientes que tienen enfermedades mentales y emocionales, así como también las enfermedades de tipo físico, tales como los asilos 
psiquiátricos y centros hospitalarios. Para Janavičienè (2010), este tipo de biblioterapia es utilizada únicamente por especialistas en el área de la medicina como una terapia adicional al tratamiento medicinal aplicado a los pacientes. Menciona que será únicamente el médico a cargo del paciente quien tomará la decisión de elegir el texto por asignarle, además de monitorear el progreso del mismo. Por otra parte, Morandi (2008) rectifica que en este tipo de biblioterapia tiene que ser siempre partícipe un profesional en el área de la salud mental pues el paciente necesitará que un especialista le ayude a canalizar sus emociones.

- Biblioterapia rehabilitacional: Si bien es cierto que este tipo de biblioterapia también se utiliza para ayudarle a los pacientes con enfermedades físicas, psicológicas o emocionales, se diferencia de la Clínica en el sentido que mientras ésta forma parte de la terapia regular del paciente, la rehabilitacional es más bien un apoyo para enseñarle al paciente cómo sobrellevar su enfermedad y adaptarse a los diferentes cambios que atravesará a lo largo de la misma, tales como posibles discapacidades a largo plazo. Se trata de darle una esperanza al paciente de que su vida continúa tal cual, por medio de lectura de apoyo y motivación (Janavičienè, 2010). Además, se diferencia de la anterior en que este tipo de biblioterapia puede ser aplicada por bibliotecólogos o por profesionales del área de la salud, ya sea en forma individual o como grupo de apoyo para el paciente. Esto último abre la posibilidad de ser trabajada también, además de en el centro hospitalario, desde una biblioteca especializada y/o la biblioteca pública.

- Biblioterapia educacional o de desarrollo de la personalidad: Tal y como lo indica su nombre, este tipo de biblioterapia corresponde a aquella que busca causar un impacto en la personalidad del paciente, con respecto a su comportamiento, su habilidad para comunicarse asertivamente con otras personas, sana convivencia, y prevención de problemas emocionales (Janavičienè, 2010).

Este tipo de biblioterapia es aplicada, preferiblemente, por personas de las áreas de servicios sociales, tales como bibliotecólogos, maestros, trabajadores sociales, etc., y puede implementarse en centros educativos, bibliotecas escolares, centros de cuido, e inclusive directamente en los hogares de los pacientes.

Al ser de carácter motivacional y recreacional, este tipo de biblioterapia permite hacer uso de un sinfín de recursos para impartirla, como lo son libros, revistas, música, películas, dibujos, danzas y juegos (Morandi, 2008), siempre y cuando el material sea el adecuado con respecto al perfil del o los participantes, y que propicie alcanzar los objetivos planteados, utilizando materiales y recursos que capten la atención de los partícipes. 


\subsubsection{Campos de acción}

La biblioterapia ofrece como gran ventaja la posibilidad de aplicarse en diversos campos de acción como una herramienta facilitadora de soluciones y mejoramiento de la actitud, por medio de la lectura y actividades complementarias a ella, pues permite trabajarse con personas que se encuentren bajo obstáculos de tipo emocional, conductual, de salud física o mental, y/o de rendimiento académico cuando son estudiantes.

De acuerdo con autores como McCulliss (2012), Tomaino (2012), Janavičienè, 2010, y Toro (2010), la biblioterapia puede desarrollarse desde el ámbito de las ciencias de la salud al ser una herramienta de ayuda para superar enfermedades físicas, desde el campo académico al trabajar con niños y adolescentes que presentan deficiencias de aprendizaje, desde el área de la psicología para colaborar con enfermos mentales y emocionales en su proceso de sanación, y desde el área de la bibliotecología, como un apoyo para reforzar valores sociales de convivencia y promover el buen comportamiento bajo diferentes métodos para contrarrestar problemas conductuales.

Se definieron entonces cuatro grandes campos de acción sobre los cuales la biblioterapia puede ser puesta en práctica, expuestos a continuación.

- Campo educativo: Aquí la biblioterapia es abordada desde centros educativos como herramienta para mejorar el rendimiento académico de los estudiantes, el comportamiento y disciplina de los mismos. Principalmente, es aplicada por docentes, trabajadores sociales, orientadores y bibliotecólogos (Cotrău, 2014).

- Campo de la salud: La biblioterapia es utilizada por parte de profesionales en el área de la medicina como lo son los médicos generales y especialistas, enfermeros, terapeutas, psicólogos, entre otros, como una herramienta tanto informativa como terapéutica, para contrapesar los efectos de las enfermedades sobre los pacientes en centros hospitalarios, prioritariamente. Se utiliza como medio de rehabilitación para que los pacientes aprendan a luchar contra su enfermedad y se mantengan motivados con una buena actitud hacia la vida (Tomaino, 2012).

- Campo psicológico: Aquí la biblioterapia es aplicada y evaluada meramente por profesionales en el ámbito de la psicología, y su desarrollo es implementado principalmente, para trabajar sobre el mejoramiento ante enfermedades mentales como la depresión (Bolitho, 2011; Smith, Floyd, Scogin y Jamison, 1997; Valverde, 2012).

- Campo bibliotecológico: Destaca el hecho de que la biblioterapia sea impartida por profesionales en el campo de la bibliotecología, desde la biblioteca, sea esta pública, educativa u hospitalaria, siempre en conjunto con grupos interdisciplinarios de profesionales como psicoterapeutas, médicos, psicólogos, educadores, entre otros (Janavičienè, 2010). 
El bibliotecólogo, al ser el profesional de la información por excelencia, es el mejor capacitado para realizar lecturas dirigidas y buscar textos que representen las situaciones y vivencias de las personas que necesitan de la biblioterapia, como herramienta de ayuda para superar problemáticas como la depresión y el mejoramiento de la conducta (Brewster, 2008; Quindemil, 2002).

\subsection{La biblioterapia y la bibliotecología}

Constantemente se ha delimitado la aplicación de la biblioterapia a áreas como la psicología o la medicina, pues mayoritariamente ha sido utilizada para aminorar conflictos psicológicos, físicos o mentales, por parte de aquellos profesionales encargados de estudiar el comportamiento humano.

Sin embargo, tanto los resultados de la investigación como la bibliografía consultada (Cotrău, 2014; Janavičienè, 2010; Tomaino, 2012) constataron que la biblioterapia puede ser aplicada también desde una perspectiva educativa, mediante una labor social en la que se trabaja la motivación del individuo con el objeto de lograr la superación en el mismo. Es bajo ésta perspectiva, donde el rol del bibliotecólogo dentro de la biblioterapia adquiere un carácter más participativo y de liderazgo en el proceso, pues al ser el profesional que posee un vínculo más estrecho con los usuarios, al estudiarlos a fondo con el fin de conocer sus necesidades, sus principales problemáticas y su entorno, será también el que mejor conoce los textos adecuados para tratar los distintos problemas que confrontan cotidianamente, orientándolos así, de la mano con la lectura dirigida y con profesionales de otras ramas del saber capaces de dar seguimiento a los avances que desarrollen las personas sometidas al procedimiento, hacia la superación de los problemas que solucionarán gracias a la biblioterapia.

Morandi (2008) presenta claramente que el trabajo del bibliotecólogo en el área de la biblioterapia será enriquecedor e irá más allá del de ser un proveedor de lecturas, si trabaja conjuntamente con los profesionales de las áreas de la educación y la psicología, principalmente. Asegura que: "el éxito [de la biblioterapia] estará en saber conjugar las habilidades del bibliotecólogo, el trabajo en equipo, la selección de la lectura y la conducción del grupo" (p. 10).

Sobre el bibliotecólogo recae, entonces, la importancia de diseñar correctamente cada sesión de biblioterapia, pues a él le corresponde la dirección del grupo de trabajo, la selección de los textos y el diseño y ejecución de dinámicas sobre las cuales se basará el método, trabajando en equipo con profesionales que evaluarán el desempeño de la terapia y la evolución y avances que obtengan los participantes de la misma. Adquiere entonces la biblioteca y el profesional de la información un rol más activo en el proceso y ejecución de la biblioterapia como tal. 


\subsection{Problemas de conducta en escolares}

Los problemas de conducta suelen ser detectados de acuerdo con la subjetividad de la persona que los observa, por ejemplo, en el caso de esta investigación, para un docente lo que puede ser un mal comportamiento, para otro podría no serlo; esta subjetividad complica mucho los problemas de comportamiento dentro de las aulas.

Sin embargo, en búsqueda de la clarificación del concepto anterior, Otero (2009) expone los problemas de comportamiento como trastornos del comportamiento, y lo conceptualiza como: "un patrón persistente y repetitivo de conducta, en el que destaca la violación de los derechos fundamentales de los demás, o las reglas o normas sociales adecuadas a la edad" (p. 1).

Esta misma autora clasifica los trastornos del comportamiento infantil en tres grupos, que son:

- Problemas emocionales, entendidos como aquellos que se relacionan directamente con la ansiedad, la angustia, la tristeza, el retraimiento social, las fobias, la concentración y el rendimiento escolar.

- Dificultades emocionales, referidas a las que se suelen manifestar en forma de síntomas muy específicos tales como tics nerviosos, miedos y patrones en comportamientos (como la succión del dedo pulgar), los cuales evolucionan favorablemente cuando son tratados.

- Problemas de comportamiento, los cuales se exteriorizan en forma de agresión, violencia, mentiras, robos, vandalismo, hiperactividad y demás conductas de carácter antisocial.

Nótese la diferenciación del nivel de complejidad entre los categorizados "problemas" con las llamadas "dificultades", siendo éstas últimas más bien pautas repetitivas en el comportamiento meramente físico, mientras que los problemas, al ser de carácter emocional y psicológico, requieren de un abordaje más amplio e intenso para ser enmendados.

Si bien es cierto que los autores dividen por diferentes categorías los problemas de conducta infantil, coinciden en que los problemas manifestados siguen siendo los mismos: la hiperactividad, la agresión, el irrespeto a la autoridad, el aislamiento, el vandalismo, entre otros.

\subsection{Cultura de paz y sana convivencia}

Actualmente, la sociedad costarricense afronta un sin número de problemas sociales como lo son la violencia, los robos, el consumo de drogas, la agresión intrafamiliar, entre otros. Este tipo de problemáticas sociales crean patrones que los niños suelen aprender en sus hogares, en sus barrios o en la sociedad como tal, los cuales imitan dentro del ámbito escolar, razón por la cual es importante contrarrestar tales conductas negativas incentivando la cultura de paz y los buenos valores en las escuelas. Los expertos en psicología infantil y juvenil, redactores del sitio web Gabinete Psicodiagnosis (2015) en Tarragona, España, afirman que 
no podemos obviar el hecho de que, en últimos años, la sociedad en general y la familia en particular, han sufrido cambios significativos en cuanto a su estructura y valores tradicionales. Ello ha supuesto también el consecuente impacto en la población infantil y juvenil. (párr. 13)

Si bien es cierto que este tipo de comportamientos infantiles son producto de las experiencias que el niño estudiante tiene en su hogar y su comunidad, también existen factores biológicos que podrían influir en que tenga un comportamiento negativo, como por ejemplo enfermedades como el déficit atencional y la hiperactividad, la ansiedad, la timidez y la impulsividad (Ministerio de Educación del Gobierno de El Salvador, 2005). De esta forma, los niños que presentan algún tipo de conducta negativa, muestran dificultades en su desarrollo educativo, tanto a nivel académico como en la sana convivencia con los docentes, otros niños o niñas, y demás figuras de autoridad escolar.

Subraya el Quinto Informe del Programa del Estado de la Nación (2015), que los problemas emocionales y de comportamiento presentes en la comunidad de estudiantes de primer ciclo en el ámbito escolar, así como el contexto social bajo el cual viven, también influyen en que se genere un bajo rendimiento académico, y por ende, un incremento en el número de niños repitentes en las escuelas del país.

Otro de los factores que influyen en que se presenten problemas de conducta entre la población escolar, siguiendo con lo expuesto en dicho informe, es la baja cobertura existente para brindar educación especial a aquellos niños que así lo requieran, pues el aprendizaje regular los abruma, y esto, genera a mediano y largo plazo, un mal comportamiento.

Entonces el contexto en el cual esté inmersa la escuela y su comunidad es de suma importancia porque influye y representa la realidad que enfrentan los niños fuera de las aulas (Chavarría y Zeledón, 2006). El problema de la violencia en las escuelas y colegios ha crecido de tal forma que se ha clasificado bajo un gran nuevo concepto, el llamado bullying, el cual es definido por el Ministerio de Educación Pública de Costa Rica (2012) como:

Una forma de acoso y violencia reiterada a lo largo de un tiempo, que puede implicar gran sufrimiento para los niños con consecuencias a veces extremas en su calidad de vida, felicidad, integración y formación. Es una forma característica y extrema de violencia escolar que requiere de una visibilización y atención especial pues requiere de un cambio cultural. (párr. 2)

Una vez señaladas cuáles son las causas que generan los malos comportamientos de la población escolar costarricense, se debe tener claro el concepto de lo que es una cultura de paz, para así buscar las estrategias necesarias y diseñar un plan de trabajo capaz de educar a los niños en valores para que mejoren su comportamiento, su integridad y su convivencia, tanto dentro, como fuera del ámbito escolar. En este sentido, Chavarría y Zeledón (2006) definen la cultura de paz "como la ausencia de violencia, de guerra o conflicto. En un sentido positivo, relacionamos la paz con el respeto y tolerancia hacia el (la) otro (a), hacia sus formas de entender y actuar en el mundo" (p. 1). 
Los mismos autores resaltan que una educación para la paz necesita una formación constante de estudiantes capaces de defender y promocionar los derechos individuales y colectivos involucrando la auto y correalización de la persona humana, tomando la construcción y reconstrucción de un proyecto socio - histórico y cultural relacionado con justicia, solidaridad, armonía, libertad y bien común.

\subsection{Estrategias de trabajo para abordar la cultura de paz y buenos valores}

La cultura de paz y los buenos valores deben ser promovidos de manera urgente dentro del Sistema Educativo Costarricense, pues de no hacerlo no será posible, a futuro, contrarrestar los efectos que han causado a lo largo de los años los comportamientos de violencia, abuso, agresión e irrespeto tanto en los niños que los manifiestan como en los que son o han sido víctimas constantes de los mismos.

Para ello existen diversas actividades pedagógicas, lúdicas y recreativas para la enseñanza de la cultura de paz entre las cuales destacan, de acuerdo con los resultados expuestos por Aguilar y Castañón (2014), las siguientes: análisis de situaciones y conductas a través de cine - foro, dinámicas de roles en donde el niño se identifique con ciertas situaciones conflictivas, juegos cooperativos que promuevan el trabajo en equipo, dramatizaciones teatrales, actividades recreativas propiciadoras de la paz, estrategias para la conciliación de conflictos habituales en las escuelas, manejo preventivo de la violencia.

\section{Metodología}

La investigación tuvo un alcance de tipo exploratorio, bajo el paradigma cualitativo, y se aplicó la investigación - acción colaborativa, definida como:

La que se realiza con la participación de personas provenientes de diferentes instituciones que deciden agruparse para reflexionar, analizar e investigar la práctica pedagógica, con el propósito de relacionar la investigación con los procesos de innovación, de desarrollo y formación profesional con interés interpretativo. (Chaves, Díaz, García, Rojas y Solís, 2010, p. 25)

Para efectos de la investigación se suplanta la parte de práctica pedagógica por la práctica pedagógica - bibliotecológica, pues ésta fue desarrollada y aplicada desde la perspectiva de profesionales en el campo de la bibliotecología, en el ambiente educativo.

La investigación - acción colaborativa se aborda bajo la modalidad práctica o hermenéutica, puntualizada por Chaves et al. (2010) como aquella a la que: 
Le interesa el proceso y no solo el producto, persigue transformación en la práctica y da cuenta de las decisiones tomadas en el proceso ... El interés se dirige a analizar las creencias y concepciones teóricas individuales, comprenderlas y actuar sobre ellas para transformarlas, en busca del mejoramiento [...] Puede contar con el acompañamiento de otras personas en el proceso investigativo: colegas, docentes universitarios, entre otros; con los que dialoga y reflexiona sobre el problema de investigación, en una relación de cooperación y mutuo respeto. (p. 28)

Los sujetos investigados incluyeron un grupo de cinco niños y una niña con edades entre los 7 y 9 años, quienes cursaron el primero, segundo y tercer grado de una escuela primaria pública costarricense, de la cual las investigadoras se reservan su nombre, como medida de protección a los sujetos que participaron de la investigación, durante el año 2015, con problemas de conducta similares, y que fueron seleccionados con base en el criterio profesional de las docentes de asistencia emocional que laboran en el centro educativo y de las bibliotecólogas a cargo de la investigación.

\subsection{Categorías de análisis}

Las categorías de análisis se determinaron durante el transcurso y progreso de la investigación, pues se construyeron, inicialmente, con base en la información que se obtuvo de la investigación teórica, y posteriormente de las observaciones y experiencias vividas durante la aplicación del servicio de biblioterapia con el grupo de infantes estudiados.

Durante el transcurso de la investigación teórica se determinaron las siguientes categorías: el servicio de biblioterpia, el comportamiento, la cultura de paz, el rendimiento, y en la investigación de campo se detectó una categoría de carácter emergente: el trabajo conjunto entre docentes terapeutas y bibliotecólogas, dentro de la cual se rescató el trabajo continuo y conjunto por parte de las docentes del área de apoyo emocional del centro educativo en cuestión, las docentes de grado y de las bibliotecólogas investigadoras del proyecto de biblioterapia, pues de no ser gracias al esfuerzo y arduo trabajo por parte dichas partes, la investigación no hubiera sido posible desde el principio.

La investigación se desarrolló a partir de los estudios de caso de seis niños de una escuela pública de educación básica, dentro del ámbito de la biblioteca escolar. El estudio de caso es conocido como "el estudio de la particularidad y de la complejidad de un caso singular, para llegar a comprender su actividad en circunstancias importantes" (Stake, 1998, p. 11).

Después del proceso de selección, los infantes fueron sometidos a 19 intervenciones de biblioterapia grupales, con una duración de una hora cada intervención, durante un lapso de dos meses comprendidos entre los meses de setiembre, octubre y noviembre del año 2015. Estas mediaciones fueron aplicadas por las bibliotecólogas investigadoras, y apoyadas con las recomendaciones de las docentes del servicio de apoyo emocional mediante el seguimiento de los resultados obtenidos de tales intervenciones con cada uno de los infantes.

Además de las intervenciones de biblioterapia aplicadas propiamente a los infantes participantes del estudio, se realizó con una intervención con las madres de familia que se presentaron con el objeto de tramitar un consentimiento informado escrito, en el que estuviesen 
al tanto del objetivo a seguir con el proyecto de biblioterapia con sus hijos, evacuaran las dudas que les surgieran con respecto al mismo, adquirieran el compromiso por llevar a los participantes a cada sesión programada, y se contara en general con su apoyo durante todo el proceso.

Se tomó la decisión de realizar intervenciones meramente grupales, en primera instancia por el factor tiempo, pues no se disponía de un periodo muy extenso para desarrollar intervenciones tipo mixtas (entre individuales y grupales), además, a raíz de las problemáticas similares que afrontaban los participantes en el estudio y luego de un debido análisis de la mano de las docentes del servicio de apoyo emocional que ayudaron en la investigación, se decidió que los mismos necesitaban desarrollar destrezas de comunicación asertiva, manejo de ira, trabajo en equipo, resolución de conflictos, entre otras más, que, para que fueran interiorizadas efectivamente, debían de tratarse desde el ámbito grupal.

Se trabajó aproximadamente una hora durante cada intervención, centrándose cumplir un objetivo por sesión basado en cada uno de los problemas que presentaba la muestra de niños en su momento, de acuerdo con los rubros expuestos dentro de las categorías de análisis del estudio, incluyendo desde el fomento de la cultura de paz y la sana convivencia, la evaluación del rendimiento tanto conductual como académico de los participantes, los problemas de comportamiento presentados por los infantes, y la aplicación del servicio de biblioterapia como tal. Para lograrlo, se efectuaron diferentes actividades de animación lectora en cada intervención, que incentivaran a la vez la cultura de paz y el amor por la lectura en los participantes, utilizando dinámicas que permitieron un seguimiento apropiado en cuanto a la promoción de la cultura de paz y sana convivencia.

Las actividades que se aplicaron a los estudiantes fueron planificadas con base en las diferentes recomendaciones encontradas luego de una búsqueda bibliográfica exhaustiva, y bajo las recomendaciones brindadas por el criterio profesional de las docentes del servicio de apoyo emocional, utilizando en gran medida técnicas como el juego, los trabajos manuales, la reproducción de cuentos en video y en audio, la narración oral, las dramatizaciones con títeres, dibujos, collages, etc., las cuales permitieron que cada uno de los infantes expresara libremente su creatividad y relacionara sus problemáticas personales con las lecturas elegidas para cada intervención.

Durante cada sesión las investigadoras llevaron un diario de campo, donde documentaron todo lo ocurrido durante su desarrollo y los resultados que fueron obteniendo con cada uno de los niños durante ese día de trabajo. Dentro de este diario de campo se tomaban anotaciones con respecto a las observaciones (tanto de aspectos positivos como negativos) percibidas en cuanto al impacto en cada uno de los infantes sobre el tema desarrollado durante cada mediación de biblioterapia, sus percepciones propias sobre los cuentos, las dinámicas y las actividades complementarias a ellos.

La biblioterapia se complementó en gran medida con el llamado sistema de promesas, con la finalidad de comprometer a los participantes a mejorar su comportamiento y así ayudarles, poco a poco, a superar las problemáticas señaladas en principio. Esta técnica se basó en las recomendaciones brindadas por Barreras y Valencia (2008), donde proponen una estrategia de conducta y compromiso, la cual consiste en mostrarle a los estudiantes tanto el compromiso que va a adquirir el docente para con ellos durante el ciclo lectivo, así como también el que los 
niños propongan también los aspectos por los cuales ellos van a comprometerse para con el profesor y para con ellos mismos, reflejando así las llamadas "promesas" con las cuales trabajaron las bibliotecólogas durante la investigación.

\subsection{Etapas de la investigación}

Al ser ésta una investigación de corte cualitativo, no fue posible seguir un procedimiento lineal específico, pues constantemente se analizaron datos nuevos que fueron apareciendo a lo largo del proceso de investigación. Así lo afirman Hernández, Fernández y Batista (2010), cuando dicen que la investigación cualitativa "no es lineal, sino iterativo o recurrente, las supuestas etapas en realidad son acciones para adentrarnos más en el problema de investigación y la tarea de recolectar y analizar datos es permanente" (p. 362).

No obstante, se siguió un hilo para realizar la investigación, donde el primer paso a seguir fue la negociación con la Directora del centro educativo para poder entrar a la escuela y trabajar con los niños miembros de la institución; seguidamente se estudió a fondo el contexto histórico, social y cultural de la Escuela y su comunidad, con el fin de conocer más a fondo el entorno de los niños sujetos de estudio; a continuación se escogieron las técnicas para la recolección de datos, en donde destacan el uso del "diario de campo", la observación y la entrevista.

Estas fueron aplicadas a las madres de los niños participantes así como también con los infantes directamente para conocer mejor el porqué de su mal comportamiento; también a las docentes del servicio de terapia emocional para conocer aspectos psicológicos de cada sujeto investigado y sus avances en el proceso biblioterapéutico, y a las docentes de grado, a cargo de educar académicamente a los infantes participantes del servicio de biblioterapia, con el fin de documentar su percepción con respecto al mejoramiento de sus problemas de disciplina y conducta en el aula. Todas las entrevistas se realizaron durante el proceso de aplicación de la biblioterapia.

Por último, se diseñaron y aplicaron los planes de intervención de biblioterapia, en los cuáles se planificaron los métodos de trabajo por utilizar, los valores por fomentar en el grupo de participantes, las lecturas por utilizar y demás técnicas complementarias de animación a la lectura, los cuales, como ya se mencionó, se publicaran posteriormente en una guía didáctica para aplicar la biblioterapia.

Una vez seleccionada la muestra del grupo de niños con quienes se trabajó, se convocó a los padres y madres de familia, o adulto encargado de los mismos, a una reunión, a la cual asistieron sólo las madres de familia, donde se les explicó la modalidad de trabajo y los objetivos planteados para la aplicación de las intervenciones de biblioterapia, además se procedió a solicitarles su permiso para contar con la participación de sus niños en el proyecto, ya que al ser menores de edad, se necesitó propiciar un debido consentimiento informado escrito, en este caso, con sus madres, pues sólo ellas se presentaron ante la convocatoria realizada.

El diseño, desarrollo y ejecución de las intervenciones de biblioterapia con los sujetos de estudio, se basó en una biblioterapia de tipo Educacional, esto porque se adecuaba más al ámbito en que se realizó la investigación: la escuela pública; además se tomó en cuenta el hecho de que el equipo interdisciplinario con el cual trabajaron las investigadoras, estuvo 
conformado por docentes de educación básica y docentes de asistencia emocional que laboran en el centro educativo, ambas profesiones del área educativa. Asimismo, debido al enfoque con que se buscaba lograr un cambio positivo en los participantes de la biblioterapia, se utilizaron recursos de lectura lúdicos y didácticos capaces de captar su atención y así obtener los resultados esperados.

Durante cada una de las intervenciones de biblioterapia, se trabajó en reforzar algún valor o buena práctica de convivencia, los cuales fueron tratados en más de una sesión con el objetivo de fortalecer más ese valor para la paz y sana convivencia en cada uno de los niños participantes, pues de acuerdo al análisis de los expedientes personales de los mismos, y bajo las recomendaciones aportadas por las docentes del servicio de apoyo emocional, habían comportamientos particulares en los que más flaqueaban los niños del estudio, tales como la ira, la agresividad, y el enojo, y por tanto debían de fortalecerse en mayor medida. Algunos de los temas abordados para la mejora de esos comportamientos, durante la aplicación de la biblioterapia en la investigación fueron: la técnica de la tortuga, el temperamento, la familia, la inseguridad, el positivismo, la ira y el enojo, la agresividad, la indisciplina, el respeto, la obediencia, el bullying, el trabajo en equipo y la comunicación.

Tal y como la afirman Aguilar y Castañón (2014), en el tema de valores es necesario educar con el ejemplo, "se debe pasar por un proceso educativo, de formación y actualización importante en tema de valores y educación para la paz, de manera de poder practicar lo que se pretende fomentar" (p. 88).

Por tanto, en cada intervención se recalcó siempre la importancia de mantener el buen comportamiento, la comunicación asertiva, el respeto y la tolerancia en general, tanto en el aula como en el ámbito familiar, buscando exponer estados de autorreflexión en cada uno de los participantes de la biblioterapia y la formación de valores sociales entre los mismos.

Como un complemento hacia una mejor terapia y la promoción de los valores y la cultura de paz, se trabajaron actividades manuales en cada intervención, en las cuales los niños debían de colorear, recortar y construir para que al finalizar cada sesión tuvieran en sus hogares diferentes materiales que les recordaran cada una de las lecciones aprendidas.

Las intervenciones grupales de biblioterapia fueron diseñadas bajo la modalidad de talleres, ya que en éstos el participante puede "sentirse en libertad de expresar sus sentimientos y puntos de vista" (Chaves et al., 2010, p. 80).

Para analizar los resultados obtenidos, se desarrollaron sesiones de discusión entre las investigadoras al finalizar cada sesión de biblioterapia, donde se condensaron ideas con el fin de realizar una conexión de temas ya abordados. Además, se efectuaron reuniones regulares con las docentes de asistencia emocional, de donde se obtenían recomendaciones y recursos de apoyo para trabajar con el grupo de niños.

Estos diagnósticos por sesión, permitieron conocer hasta qué punto los participantes aprendieron sobre los temas desarrollados. De igual manera se mantenía un constante contacto con las maestras, terapeutas y madres de familia de los diferentes infantes que formaron parte del proceso, con el objetivo de conocer si presentaban algún cambio. 
El orden en que se diseñó el abordaje de los problemas que se tocaron en cada sesión, favoreció a reforzar comportamientos y técnicas que permitieron a los infantes tener actitudes asociadas a una cultura de paz.

Las investigadoras planean publicar una guía didáctica, donde se explique a detalle cuáles fueron las dinámicas, lecturas, materiales de apoyo, técnicas de animación lectora, y demás instrumentos con los que se trabajó cada una de las intervenciones de biblioterapia, así como también los planes de trabajo para el abordaje de cada uno de los valores y buenas prácticas de convivencia que se fomentaron en el grupo.

\section{Análisis de los resultados}

Es importante mencionar que todos los infantes siguieron con las intervenciones de biblioterapia hasta el final, excepto uno de ellos, a quien llamaremos "José", quien se retiró del programa durante las primeras intervenciones, pues no mostró un interés genuino por la actividad y no le gustaba del todo la lectura.

Para desarrollar el análisis, se utilizó el método de triangulación de datos, el cual "consiste en contrastar e interpretar los datos, considerando diversas fuentes, técnicas de recolección, actores y referentes teóricos." (Chaves et al., 2010, p. 85).

Resalta el hecho de que al ser ésta una investigación de tipo cualitativa, hubo gran flexibilidad en cuanto a la modificación de las categorías de análisis que se propusieron en un principio, permitiendo adaptarlas según la información que se fue obteniendo a lo largo del proceso investigativo. A partir de esto surge una categoría de análisis emergente, referente al trabajo interdisciplinario durante todo el proceso del servicio de biblioterapia, por parte de las profesionales en bibliotecología, ya que una de las investigadoras labora para la biblioteca escolar de la institución, y las docentes del servicio de apoyo emocional a los escolares del centro educativo, y por supuesto, con las docentes de grado de los infantes que formaron parte de la investigación, permitiendo un análisis más detallado de las reacciones de los mismos durante la aplicación de la biblioterapia, posteriormente.

A continuación, se resumen los resultados de la investigación por categoría de análisis.

\subsection{Servicio de biblioterapia}

El servicio de biblioterapia se llevó a cabo desde la biblioteca escolar, a través de la animación a la lectura, donde se utilizaron diferentes materiales bibliográficos para promover un efecto positivo en el comportamiento de los participantes, a su vez la biblioterapia se convierte en una opción diferente para trabajar con aquellos chicos que presenten desordenes de conducta tanto en el ambiente escolar como en el hogar, tal y como y se adaptó con los niños que se trabajó durante la investigación. Esto logra posicionar a la biblioteca escolar y al bibliotecólogo como agentes de cambio en el entorno escolar, pues ya no es solo aquel lugar para préstamo de libros sino que, es punto de encuentro en la escuela donde se fortalecen los valores, se promueve la cultura de paz y se fortalece la animación a la lectura. 
Según la experiencia se considera que la biblioterapia es un buen método para fomentar la lectura en la población participante, la clave está en que se planeen y ejecuten las actividades complementarias de una manera atractiva y divertida para los niños, pues esto es lo que les llama la atención.

Mantenerlos activos con dinámicas y actividades hacen que los participantes muestren un alto grado de interés por ir a cada sesión. Aunado a estas actividades se incentiva y fortalece la animación a la lectura, entonces al terminar el proceso el niño no solo habrá aprendido y/o reforzado valores para la sana convivencia, sino que también puede adquirir el hábito de ser lector.

En cada una de las sesiones se trabajó con un cuento, ya fuera este en texto, video o audio, en el cual su idea principal fue el fortalecimiento de un valor y/o actitudes para la sana convivencia. Como parte de la biblioterapia, el cuento se vio acompañado de alguna actividad complementaria, que permitió fortalecer lo aprendido durante la sesión, se realizaron actividades como: construcción de manualidades, observación de videos, mesas redondas, creación de dibujos, colorear como medida de relajación terapéutica, técnicas de relajación y compromisos de buen comportamiento. Además, cada sesión de la biblioterapia, aunque tuviera un argumento diferente, fue contemplada dentro de dos grandes temáticas, el comportamiento y la cultura de paz.

En este caso la biblioterapia de tipo educacional lo que pretende es contribuir en la formación de valores de los y las estudiantes y así promover la cultura de paz por lo tanto para abordar dicha temática se realizaron diversas actividades como por ejemplo: Elaboración de un collage sobre la familia, antifaces relacionados al cuento, el pulpo con lentejuelas, elaboración de dos contratos de obediencia, construcción de un gusano sobre lo que es el bullying, entre otros.

\subsection{Comportamiento}

La categoría de análisis de comportamiento se enfocó principalmente en el manejo de la ira, el enojo y la agresividad, debido a que los niños participantes presentaban conductas agresivas y explosivas, tanto en sus hogares como en la escuela, y debido a esto eran atendidos en el servicio de apoyo emocional y de conducta de la institución educativa. El tema se trabajó por medio de intervenciones cuyo objetivo era aprender a manejar tales emociones. También se abordó la enseñanza de otros valores, relacionados con el comportamiento, como el respeto, la obediencia, la disciplina, la familia, la comunicación, el bullying, el positivismo y el temperamento.

En algunas ocasiones se trabajó algún valor o tema en más de una sesión con el objetivo de reforzarlo en cada uno de los niños participantes, de acuerdo con el análisis previo de cuáles eran los comportamientos en que los mismos flaqueaban en mayor medida, y bajo las recomendaciones aportadas por las docentes del servicio de apoyo emocional.

La biblioterapia no solo permite que los niños se identifiquen con los personajes del cuento y de este modo puedan asociarlos y buscar una solución positiva a tales circunstancias, sino que permite que por medio de la lectura los participantes puedan conocer técnicas capaces de utilizar en el caso de que se encuentren enojados. 
De lo anterior se destaca el hecho de que las actividades, dinámicas y juegos complementarios incentivaron la expresividad y apertura emocional de los infantes participantes, permitiéndoles despegarse de sentimientos de resentimiento y malestar que venían arrastrando de sus experiencias ante situaciones y conflictos vividos en el pasado.

El comportamiento de los niños siempre fue una prioridad en cada sesión, por lo tanto, siempre se buscó incentivar en ellos actitudes capaces de llegar a soluciones en una cultura de paz, aunque el grupo de niños que inició no terminó completo debido a que uno de ellos desertó pues afirmó que le parecía muy aburridas las sesiones debido que en todas se leía un cuento. De lo anterior, se observó que antes de someter a cualquier persona a un proceso de biblioterapia, es necesario interesarla primeramente en la lectura, pues si la persona se cansa de leer, puede no conseguirse el impacto esperado, y por supuesto, la renuncia de la persona a continuar en el proceso, como en este caso.

En contraposición a lo anterior, las docentes por su parte se mostraron muy satisfechas con los cambios que notaban en sus estudiantes a medida que se avanzaba en el proceso biblioterapéutico. Las maestras destacaron cambios en el comportamiento después de las intervenciones como una actitud muy positiva, grandes cambios en su autoestima, aumento de motivación, incluso en algunos de los estudiantes se reportaron pequeños cambios, como una mayor tranquilidad y disminución en sus actitudes violentas, e incluso uno de ellos adquirió un sentido de responsabilidad mayor dentro de su clase.

Las madres de familia también notaron cambios positivos en el comportamiento de sus niños. Una de ellas mencionó que su hijo se veía muy motivado para asistir a los talleres y que también a ella le llamaba la atención cuando se realizaban manualidades y tenían que seguir un proceso en las casas, como por ejemplo la dinámica de la pulsera del enojo. Alegó también que su hijo es diferente desde que asistía a los talleres de biblioterapia, esto porque presenta un manejo más adecuado de emociones en situaciones de enojo. La madre del niño que se salió del taller, afirmó que aunque su hijo comentó que le aburrían los talleres porque siempre se leían cuentos, en las pocas sesiones que participó llegó contando lo sucedido a su casa. Además, recordaba personajes y actitudes de los protagonistas de las historias frente a circunstancias difíciles, haciendo análisis de lo sucedido en los cuentos. Las madres manifestaron un gran agradecimiento al notar las mejoras en la conducta de sus hijos.

Con respecto a la opinión de las docentes entrevistadas, coincidieron en que uno de los niños en especial, aprovechó al máximo las intervenciones de biblioterapia, pues logró un cambio muy grande en la forma en que socializaba con otros compañeros de clase, mejorando su disposición a la hora del juego y mostrando una mayor apertura para compartir con sus nuevas amistades; además el mejoró la confianza en sí mismo. Se comentó, además, que otro de los niños ha cambiado un poco su actitud, pues sigue intentando llevarse mejor con sus compañeros de clase; manifestaron que se evidenció un ligero cambio en otro de los infantes, porque se observaba más quieto, pensativo y con un poco más de responsabilidad en la clase. Se reportó que en otro de los casos trabajados se presenta más alegría y motivación para asistir a las sesiones y que se logró mejoras el área de convivencia con los demás compañeros. De acuerdo con las educadoras los niños del estudio presentaron en general una mayor motivación con respecto a las situaciones cotidianas y al ambiente escolar. 


\section{3.}

\section{Cultura de paz}

En general, durante las sesiones de biblioterapia los participantes mostraron mucho interés, sin embargo, algunas características propias de sus conductas, ya citadas anteriormente, hacían que se distrajeran con facilidad, se molestaran en ocasiones, e inclusive llegaran a desafiar a las investigadoras. No obstante, durante todo el proceso se tuvo gran insistencia en cuanto a la formación de valores para la paz y la sana convivencia, por medio de los talleres, los cuentos utilizados, y todas las actividades complementarias a la lectura que fueron aplicadas, que poco a poco fueron transformando las conductas negativas en positivas.

De acuerdo con los resultados de la investigación se percibió que para los participantes, los estímulos positivos son de gran importancia, sobre todo cuando se busca fortalecer una cultura de paz. Para ello se utilizaron diferentes métodos para la motivación de los niños, como lo fueron la aplicación de un sistema de recompensas durante las sesiones de biblioterapia, como por ejemplo la premiación de las buenas acciones y comportamientos por medio de stickers, dando las felicitaciones ante los buenos comportamientos (es decir, no solamente regañar cuando se hacía algo malo), y trabajando de la mano con las docentes de grado y las madres de familia, para que continuaran con la motivación constante desde el aula y en el hogar, y así causar un mayor impacto en los niños.

Al contrastar el comportamiento inicial de los sujetos de estudio, de acuerdo con sus expedientes, con el resultado obtenido al finalizar la biblioterapia, destacó uno de los niños en particular, pues inicialmente llegó con un nivel bajo de tolerancia y alta frustración, y al finalizar las sesiones el niño no presentaba más esta conducta, por tanto, se le consultó a su maestra de grupo, quien indicó que el niño ha superado ya esa etapa y que a nivel escolar no causa ningún problema. Gracias a ello fue dado de alta en el servicio de apoyo emocional y de conducta.

Algunos infantes entendían mejor las instrucciones de las actividades que otros, ejemplo de ello se demostró durante la actividad escrita del semáforo, que consistía en señalar, por medio de los colores del semáforo, cómo reaccionarían ante una situación " $X$ ", donde el color rojo representaba la violencia, el amarillo el enojo controlado, y el verde el estado de calma y la utilización del diálogo. Para la mayoría de los niños fue fácil la identificación, sin embargo para "Marco" no lo fue, ya que al niño le costaba entender qué era lo que se decía, cuando se exponían las situaciones " $X$ " de la dinámica, por tanto requirió un poco más de explicación y ayuda.

Al finalizar las sesiones se les consultó a las docentes de grado sobre el comportamiento de los niños durante, y especialmente, después de las sesiones a lo que todas a través de un cuestionario indicaron la mejoría en cada niño y niña, en el tema de la sana convivencia de ellos con sus respectivos grupos. Se detallan los siguientes datos:

- Maestra de "Pablo": "Mostró una actitud positiva y alegre."

- Maestra de "Juan": "Mostró mayor responsabilidad en el salón de clase."

- Maestra de "Ana": "Su actitud cambió un poco, tratando de llevarse mejor con sus pares". 
- Maestra de "Esteban": "Después de las sesiones el niño se mostró un poco más motivado con respecto a las situaciones cotidianas del ambiente escolar".

- Maestra de "Marco": "El niño mostró más apertura con respecto a sus compañeros".

\subsection{Rendimiento académico}

Sólo uno de los niños no logró ganar su año escolar: "Marco". Es necesario aclarar que a él se le aplicaba adecuación curricular, pues presentaba problemas de aprendizaje y, sumado a esto, lamentablemente el apoyo familiar es escaso. A pesar de esto su maestra si reportó un mejoramiento en su conducta dentro del aula.

En el caso de "Juan", era un niño que había sido trasladado de otra escuela y sus calificaciones mejoraron en gran manera, por ejemplo, según el perfil de salida del niño, en el primer trimestre en la materia de Estudios Sociales llevaba un promedio de 55 mientras que en el tercer trimestre su nota fue de un 80; en el caso de inglés, en el primer trimestre su nota fue de un 50 y en el tercer trimestre su promedio fue de 92.

"Pablo", otro de los niños, quién cursa el primer grado, no recibe calificaciones numéricas, sino que se describen sus logros en su expediente académico, de acuerdo con el modelo de evaluación de Primer año del MEP. La maestra anota en su evaluación del segundo trimestre: "su conducta es buena siga esforzándose por mejorar", mientras que en el tercer trimestre le hizo la siguiente observación: "Lo felicito mejoró mucho su conducta, siga así."

Otra mejora la obtuvo "Ana". Su maestra la describe como una niña muy inteligente y capaz, la cual concluyó su año con muy buenas calificaciones y mejoró en su comportamiento, por lo tanto fue dada de alta en el servicio de apoyo emocional. "Esteban", aunque fue el niño con el temperamento más fuerte y retador, también mejoró sus calificaciones y, al igual que los demás, ganó el curso lectivo.

\subsection{Trabajo conjunto docentes terapeutas - bibliotecólogas}

En el inicio de la investigación se definieron las categorías de análisis que se mencionaron anteriormente. Sin embargo, durante el proceso de análisis y recopilación de datos, surgió como categoría emergente el trabajo en conjunto entre las docentes encargadas del servicio de apoyo emocional, las docentes de grado y las bibliotecólogas investigadoras, que se desarrolla a continuación.

La investigación fue trabajada de principio a fin con el apoyo complementario de las docentes de servicio de apoyo emocional que laboran en el Centro educativo, así como con las docentes de grado de cada niño.

A medida que se desarrollaban las intervenciones de biblioterapia con los niños participantes, se dio a la vez un trabajo estrecho entre las partes mencionadas con anterioridad, que realizaron un gran esfuerzo continuo, en el que se buscaban herramientas para mejorar la disciplina de los niños durante las intervenciones, actividades que fueran atractivas y a la vez 
educativas para los mismos, se organizaban horarios de trabajo con las docentes de grado para evaluar y dar seguimiento a los estudiantes, se daban inducciones cortas a las docentes de grado para que ellas aprendieran a controlar el comportamientos de los participantes en el aula y para que ellas cumplieran un papel como agentes reforzadoras de los valores y buenos comportamientos que se enseñaba a los mismos durante la biblioterapia.

Se establecieron reuniones fuera del tiempo laboral con las docentes terapeutas del servicio de apoyo emocional para compartir experiencias y consejos sobre la aplicación de la biblioterapia, y a la vez ir evaluando los resultados logrados en los niños participantes, entre otras actividades varias que demandaron tiempo y recursos de ambas partes.

Las docentes del servicio de apoyo emocional señalan que a medida que avanzaban las intervenciones de biblioterapia, y por supuesto, con la asistencia regular brindada por ellas mismas a cada uno de los infantes durante sus sesiones terapéuticas, los mismos se mostraban cada vez más motivados por asistir a los talleres de biblioterapia, y estuvieron muy anuentes a los consejos y pautas a seguir que se trataban dentro de los mismos.

Por su parte las docentes de grado manifestaron también que sus estudiantes poco a poco habían ido mejorando, en el sentido de que adquirieron un mayor sentido de responsabilidad en cuanto a sus quehaceres académicos, además de que ya no peleaban tanto con sus compañeros de clase.

Dentro de esta categoría, cabe resaltar también, el papel que cumple la biblioteca escolar como agente de cambio para este tipo de población estudiantil, quienes presentan problemas de comportamiento y disciplina constantes, los cuales necesitan ser corregidos por medio de actividades que no sean únicamente terapéuticas desde un sentido psicológico, que es el área en que se enfatiza el servicio de apoyo emocional de las escuelas costarricenses, sino que también sean abordados desde un punto de vista educativo y lúdico - recreativo, en donde el niño pueda expresarse e identificarse por medio de cuentos de reflexión, y de actividades y juegos de convivencia que le permitan auto corregirse potenciando el sentimiento de empatía para con las figuras de autoridad, seres queridos, familiares y compañeros.

Por último, es importante afirmar que este trabajo en conjunto logró unir conocimientos de tres profesionales en diferentes áreas, por ejemplo el bibliotecólogo según su formación es el encargado de buscar los textos adecuados a cada caso y aquellas actividades de animación lectora, como promotor de cultura. La docente de grado conoce a los niños en su ambiente escolar y sabe cómo son las relaciones interpersonales con sus similares y, por último, la docente de terapia emocional es quien conoce más a profundidad los casos, así como aquellos factores que más influyen en la vida de los niños. Esto consintió que entre todos se diera un flujo de información constante, permitiendo desde el inicio la escogencia de los niños, así como en la conclusión del proyecto, conocer cuál era el avance de los mismos por medio del sistema de triangulación de datos requerido para el análisis de la información. 


\section{Conclusiones}

En general, los resultados obtenidos de la aplicación de las intervenciones de biblioterapia con un grupo de infantes seleccionados que presentaban problemáticas similares en cuanto a comportamiento, disciplina y agresividad, dentro del centro educativo, enfocadas hacia la formación de una cultura de paz, sana convivencia y buenos valores sociales, fueron muy positivos, pues poco a poco los participantes han adquirido una mejor actitud en su comportamiento, especialmente hacia las docentes de la escuela y hacia sus madres, como así lo han expresado ellas mismas mediante las entrevistas evaluativas del proceso.

Lo mismos resultados se pudieron constatar durante la ejecución de los talleres de biblioterapia, donde los niños participantes se mantuvieron interesados y abiertos a cambiar sus malos comportamientos, y realmente se notaron grandes cambios en ellos al finalizar las intervenciones.

El servicio de biblioterapia aplicado se ajustó a un lapso de aproximadamente dos meses. Durante este tiempo de trabajo los participantes dieron sus primeros pasos hacia una mejoría, sobre la cual deberán seguir trabajando junto con sus docentes y familiares. Si en un periodo de dos meses se obtuvieron los avances ya mencionados, se esperaría que al ejecutar proyectos de biblioterapia durante un mayor tiempo, se obtengan aún resultados más sólidos y positivos en los participantes.

Las sesiones trabajadas de una manera creativa dejan huella en los participantes, sin embargo, es necesario que el tiempo de terapia sea mayor, preferiblemente de una duración de un año de ciclo lectivo, pues como ya se explicó, debido a que se debía concluir esta investigación y por el cierre del curso lectivo, se trabajó la biblioterapia durante dos meses solamente. De ahí que los niños en ocasiones presentaran conductas negativas perturbando el ambiente escolar y familiar, mientras estuvieron participando de la biblioterapia, por tanto, para generar mayor impacto se recomienda extender el plazo por trabajar en la biblioterapia.

En general, cinco de los participantes del servicio de biblioterapia cumplieron con el objetivo propuesto en el principio de la investigación, algunos en mayor grado que otros, pero con resultados positivos que poco a poco han ido mejorando su actitud, su comportamiento social, e inclusive su rendimiento escolar, poniéndole más empeño a sus deberes escolares y a su aprendizaje. Prueba viviente de ello se constata en "Pablo", quien se recuperó de tal forma que inclusive fue dado de alta del servicio de terapia emocional, posterior a su participación de las intervenciones de biblioterapia.

Entre las situaciones observadas, destacó el hecho de que no a todos los infantes les gusta la lectura, pues en ocasiones se mostraban algo aburridos durante el momento del cuento en las intervenciones de biblioterapia. Del proceso se dedujo también que al trabajar con niños que presentan problemas de disciplina, es necesario trabajar las actividades complementarias a la lectura en formas muy dinámicas, a través de juegos, actividad física y manualidades, pues al presentar cierta hiperactividad, pierden la concentración fácilmente lo que genera que se distraigan y se aburran. 
Así mismo, se concluyó que al trabajar conductas disfuncionales es necesario que los niños se atiendan tanto de manera individual como grupal, debido a que había ocasiones donde fue más provechoso trabajar de forma individual porque permitió una mejor interiorización que cuando se abarcaba en grupo.

La hora de la lectura conforma la parte más importante de toda aplicación de biblioterapia, por lo que ésta debe apoyarse en gran medida con diferentes técnicas de animación lectora, en donde no se trate solamente de leer o narrar un cuento, sino de hacerlo divertido y diferente, con el soporte de materiales diversos como dibujos, videos, títeres, juegos lúdicos y recreativos, y demás actividades que pongan a trabajar la concentración y la creatividad del infante, así como también el generar que la lectura cumpla el impacto deseado en el niño o niña.

Se trabajó la biblioterapia según las recomendaciones de expertos en el área de terapia emocional, consultados para efectos de la investigación, sobre las estrategias que se pueden utilizar para aplicar este servicio, durante la propia aplicación de la biblioterapia por parte del equipo investigador. Fue notable que no todas estas estrategias de animación a la lectura resultan exitosas para lograr los objetivos deseados en un principio, por ejemplo, casos en los cuales el grupo de participantes se dispersó en gran medida, teniendo mucha indisciplina, lo que dificultó extremadamente el flujo de trabajo planeado para esas sesiones.

El caso específico de la investigación detectó que el bibliotecólogo escolar puede llevar a cabo y liderar un proceso de aplicación de biblioterapia de forma exitosa, liberándolo del mito de que solamente los profesionales del área de la salud pueden desarrollarla, y convirtiendo a los bibliotecólogos de las bibliotecas escolares en candidatos potenciales para brindar el servicio de biblioterapia en los centros educativos costarricenses, señalando también la importancia de recibir el apoyo necesario por parte de especialistas en otras áreas del saber, que también forman parte del personal docente de estas instituciones, y quienes por supuesto brindan las herramientas, evaluaciones y recomendaciones necesarias a lo largo del proceso. Los bibliotecólogos del sistema de educación escolar costarricense cuentan con formación profesional pertinente para trabajar la lectura de forma divertida, amena, interesante y atractiva para los infantes, por medio de diferentes herramientas y técnicas para la animación lectora.

Dentro del sistema escolar costarricense la biblioterapia aplicada por bibliotecólogos es más que posible, siempre y cuando reciban capacitación adecuada; hay orientadoras, docentes especializados en atención de terapias emocionales, psicólogos, educadores, entre otros, quienes de forma conjunta pueden prestar este servicio a los y las estudiantes.

Se evidenció cómo la biblioteca escolar actúa como un agente de cambio, promoviendo la cultura de paz dentro del estudiantado, por medio de programas de lectura, actividades y juegos que los involucren en el buen camino y en la auto reflexión, sin embargo, aún existe gran desconocimiento en el país sobre el rol de la biblioteca y el profesional de la información, motivo por el cual se vuelve necesario empezar desde las escuelas, en la infancia, a incluir la biblioteca escolar dentro de su cultura y la educación cotidiana, e ir fortaleciendo poco a poco la cultura de paz dentro del ámbito escolar costarricense.

Destaca el hecho de que las bibliotecólogas investigaron sobre la teoría de la biblioterapia y sobre las estrategias necesarias para poderla aplicar, además de que consultaron con 
profesionales del área de terapia, específicamente las docentes que laboran en la escuela en que se realizó la investigación, sobre cómo llevar a cabo este programa de la mejor manera posible. Por lo tanto, aprender a aplicar la biblioterapia requiere un gran esfuerzo y se considera que al bibliotecólogo, en Costa Rica, no se le otorga una capacitación especializada en la temática que le permita conocerla y aplicarla con facilidad.

Además, la aplicación de la biblioterapia en el centro educativo requirió una inversión económica por parte de las investigadoras, pues se necesitó fabricar material constantemente para llevar a cabo de forma exitosa las intervenciones planificadas, sin mencionar otras inversiones para motivar a los participantes para el cierre del proyecto y las debidas recompensas con que se premiaban en ocasiones, bajo el sistema de recompensas ya mencionado en el apartado metodológico.

Señalar el hecho de que la biblioterapia, en el campo de la bibliotecología, es más un apoyo socioeducativo, que médico y/o psicológico, pues en lo que se trabaja es en establecer vínculos estrechos con los participantes: conocerlos, escucharlos, aconsejarlos, recrearlos, colaborando en el cumplimiento de sus metas, que al final del proceso consiguen generar un impacto positivo en el participante, que le permite salir adelante en pro de una mejora continua.

A modo de cierre las investigadoras dejan abierta la oportunidad de indagar más sobre la biblioterapia a otros investigadores, para que se aplique y se estudie más a fondo en futuros proyectos de trabajos finales de graduación, de forma tal que se descubran nuevas técnicas para implementarla, se enfoque en otros niveles educativos de la educación costarricense y por supuesto, que se le brinde la ayuda necesaria a aquellas personas que necesitan una mano amiga y que pueden encontrar serenidad y sanidad en las valiosas páginas de los libros.

\section{Agradecimientos}

A la Directora de la Escuela pública costarricense en que se realizó la investigación, a las docentes que formaron parte del equipo interdisciplinario, a los niños que participaron en la investigación y a sus madres, pues sin el apoyo de todos ellos esta no hubiera sido posible.

\section{Referencias}

Aguilar, M., y Castañón, N. (2014). Propuesta actividades y estrategias pedagógicas que promuevan una cultura de paz y capacidad de resolución de conflictos en instituciones educativas venezolanas. Revista de Comunicación de la SEECI, 17(34), 3-94.

Barreras, M., y Valencia, P. (2008). Manual de apoyo para docentes: estrategias de manejo conductual en el aula. Chile: Fundación Paz Ciudadanía. Recuperado de http://ww2.educarchile.cl/UserFiles/P0037/File/Inspector/Paz\%20ciudadana\%205\%20Manual Mane jo de Aula Docentes Final 17.11.08.pdf

Bolitho, J. (2011). Reading into wellbeing: bibliotherapy, libraries, health and social connection. APLIS, 24(2), 89-90.

Brewster, L. (2008). Medicine for the soul: bibliotherapy. APLIS, 21(3), 115-119. 
Chavarría, E., y Zeledón, M. (2006). Antología del taller: cultura de paz y resolución de conflictos. Costa Rica: Ministerio de Educación Pública.

Chaves, L., Díaz, M., García, J., Rojas, G., y Solís, N. (2010). Investigación-acción colaborativa: Un encuentro con el quehacer cotidiano del centro educativo para su transformación. San José, Costa Rica: INIE.

Cotrău, S. (2014). Child development through bibliotherapy. Journal Plus Education, 10(1), 239-250.

Gabinete Psicodiagnosis. (2015). Problemas conducta infantil. Recuperado de https://psicodiagnosis.es/areageneral/ciclo-evolutivo/problemas-conducta-infantil/index.php

Gómez, K. (2011). La biblioterapia como práctica profesional del bibliotecólogo (tesis de licenciatura inédita). Universidad Central de Venezuela, Venezuela.

Handbook of Non Drug Intervention Project Team. (2013). Bibliotherapy for depression. Australian Family Phisician, 42(4), 199-200.

Hernández, R., Fernández, C., y Baptista, P. (2010). Metodología de la Investigación (5ª ed.). México: McGraw Hill.

Janavičienè, D. (2010). Bibliotherapy process and type analysis: review of possibilities to use it in the library. TILTAI, (4), 119-132.

McCulliss, D. (2012). Bibliotherapy: Historical and research perspectives. Journal of Poetry Therapy, (1), 23-38.

Ministerio de Educación del Gobierno de El Salvador. (2005). Manejar problemas emocionales y de comportamiento en las aulas: una guía didáctica para Directores y Docentes. Recuperado de http://www.inclusioneducativa.org/content/documents/Mod4-EducacionInclusiva.pdf

Ministerio de Educación Pública. (2011). La Convivencia en los Centros Educativos en Costa Rica. Módulo 2. Promoción y Prevención. Socioanálisis de América Latina. Recuperado de https://www.unicef.org/costarica/docs/cr pub Guia Estrategia Convivencia en Centros Educativo $\underline{\text { s.pdf }}$

Ministerio de Educación Pública. (2012a). Acoso, matonismo, bullying y el ciberbullying en los centros educativos de secundaria. Recuperado de http://www.franco.ed.cr/wpcontent/uploads/2014/07/BULLYING.pdf

Ministerio de Educación Pública. (2012b). Programa Convivir. Recuperado de http://www.mep.go.cr/programas-y-proyectos/programa-convivir

Morandi, V. (2008). La logoterapia y la biblioterapia: descubriendo el sentido de la vida a través de la lectura. Uruguay: Universidad de Montevideo.

Otero, M. (2009). ¿Cómo afrontar los problemas de comportamiento desde la escuela? Cuadernos de Educación y Desarrollo, 1(9). Recuperado de http://www.eumed.net/rev/ced/09/nom.htm

Programa del Estado de la Nación. (2015). Quinto informe del Estado de la Educación. San José, Costa Rica: Autor. Recuperado de http://www.estadonacion.or.cr/educacion2015/\#capitulos 
Quindemil, E. (2002). Proyecto de aplicación de Biblioterapia infantil en la Biblioteca Pública Municipal de Güines. Revista de Ciencias Médicas, 8(1). Recuperado de http://revcmhabana.sld.cu/index.php/rcmh/article/view/88/html

Salinas, W. (2009). La Biblioterapia como complemento en el ámbito educativo. Ponencia presentada en el $12^{\circ}$ Congreso Internacional de Promoción de la Lectura y el Libro, Fundación El Libro, Buenos Aires, Argentina.

Silva da, T. M. (2011). ¿Como o bibliotecário pode se inserir nas atividades de leitura como biblioterapia? Recuperado http://monografias.ufrn.br:8080/jspui/bitstream/1/181/1/TaiseMS_Monografia.pdf

Smith, N. M., Floyd, M. R., Scogin, F., y Jamison, C. S. (1997). Three-Year Follow-Up of Bibliotherapy for Depression. Journal of Consulting and Clinical Psychology, 65(2), 324 - 327.

Stake, R. (1998). Investigación con estudio de casos. Madrid, España: Morata.

Tomaino, V. (2012). Biblioterapia: una nueva herramienta para el profesional bibliotecario. Ponencia presentada en la II Jornada "Temas actuales en bibliotecología", Centro Médico Mar del Plata, Argentina. Recuperado de http://eltopodealejandria.blogspot.com/2012 0101 archive.html

Toro, C. (2010). Biblioterapia y tus necesidades. Horizontes, (102-103), 39-40.

Tukhareli, N. (2011). Bibliotherapy in a Library Setting: Reaching out to Vulnerable Youth. Partnership: the Canadian Journal of Library and Information Practice and Research, 6(1), 1-19.

Valverde, V. (2012). Libros que curan. Revista Equilibra, (29). Recuperado de http://www.revistaequilibra.com/189-Libros-que-curan.html 


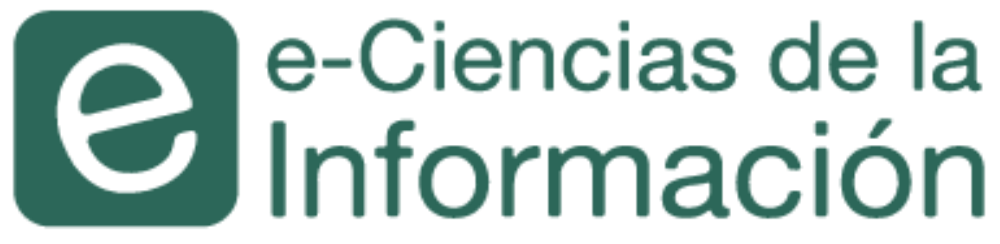

¿Desea publicar su trabajo? Ingrese aquí

O escríbanos a la siguiente dirección: revista.ebci@ucr.ac.cr

(두)(2)

\section{1}

Origen: respuesta a una necesidad

En el año 2011, la Escuela de

Bibliotecología y Ciencias de la Información (EBCI) de la Universidad de Costa Rica (UCR) reconoció la importancia de crear nuevas y mejores alternativas para difusión de la investigación. e-Ciencias de la Información es la respuesta a un contexto actual marcado por una mayor apertura, flexibilidad, y rigurosidad en la verificación de los datos y su procesamiento.

\section{3}

\section{Revista de la UCR}

e-Ciencias de la Información es una revista científica que aborda las nuevas temáticas de desarrollo e investigación en las Ciencias de la Información, en el ámbito nacional e internacional. Así, colabora significativamente en el progreso de esta disciplina. Por sus parámetros de calidad, pertenece al grupo de las revistas más importantes de la UCR y se encuentra ampliamente indizada en los importantes catálogos.

\section{En la actualidad}

\section{Posicionamiento internacional}

Se encuentra en el Cuartil A del UCR Índex para el 2017, posicionándola como una de las mejores revistas de la Universidad de

Costa Rica, un reflejo claro y conciso sobre su calidad y trascendencia en el área apoyado por otros hitos como su ingreso a Scielo, DOAJ, Latindex y otros.
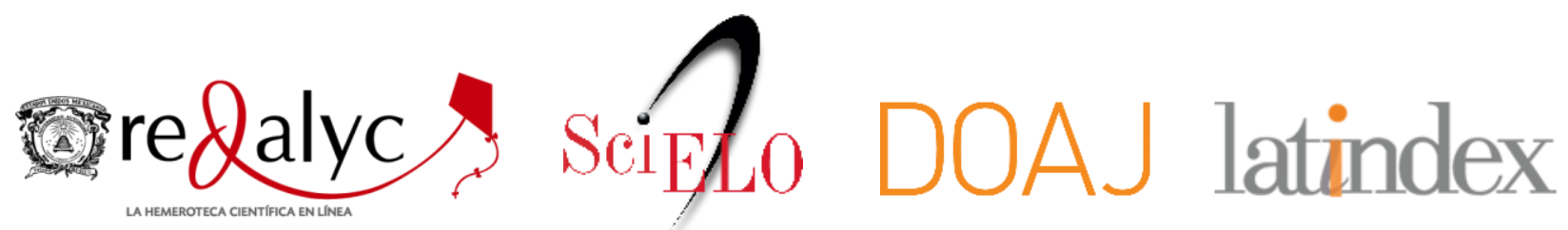

e-Ciencias de la Información está indexada en los catálogos más prestigiosos.

Para conocer la lista completa de índices, ingrese aquí 